\title{
Optimum Design for Adaptive Equalizers based on Fractional Lower-Order Statistics in non-Gaussian Environment
}

\author{
M. Rupi, and E. Del Re. \\ LENST Laboratory - Department of Electronic Engineering \\ Facoltà di Ingegneria, Università di Firenze \\ Via di Santa Marta 3, 50139 FIRENZE, Italy
}

\begin{abstract}
Adaptive channel equalization is an effective tool for the antenna receiver for estimating the information sequence in severe interference backgrounds. Therefore, the problem of linear channel distortion has been the focus of considerable research in the signal processing and communications conmunities.

The choice of antenna receiver structure is a challenge for next radio communication systems, because from this will depend most of the improvement will be possible with efficient dsp devices. The two possible streets are the use of omnidirectional antennas or antennas array.

Our paper presents innovative adaptive equalizers working on in different not necessary Gaussian environmental conditions.
\end{abstract}

\section{Introduction}

The rapidly increasing demand for personal mobile communications has created an avid interest in innovative services and in new robust signal processing techniques, which could better protect and recover the transmitted information sequence. Extensive research effort has been directed towards propagation impairment attenuation and interference mitigation in order to make efficient use of the finite spectral resources of a physical channel.

The atmosphere and the ocean, playing the role of the physical channel in wireless communications, can cause signal distortion due to finite bandwidth and signal lading due to multipath propagation. In the past, an abundance of adaptive linear equalizers have been proposed, which optimize an appropriate performance index to adaptively compensate for the effects of the channel [8]. A large portion of the proposed methods assumes the availability of a training sequence at the receiver for the purpose of initially adjusting the equalizer coefficients. However, in applications such as multiuser communication networks and mobile communication systems, it is desirable for the receiver to be able to equalize the channel without the use of a training sequence (self-recovering or blind equalization) [2].

During the $90 \mathrm{~s}$, a great interest in wireless communication applications including blind equalization, source separation, and antenna beamforming has driven research into the development of fast and robust algorithms. Nowadays, one of the most studied and implemented in practice blind cqualization methods is the Constant Modulus Algorithm (CMA). The CMA is based on a criterion that penalizes deviations of the modulus of the equalized signal away from a fixed value determined by the source alphabet. The first major study of the CMA and its properties was performed by Treichler et al. $[10,11]$ who analyzed the capture and lock behavior of baud-spaced CMA using an omnidirectional antenna receiver. A further study was done by [1] where was analyzed the algorithm performance using arrays of antennas at the receiver station.

By and large, the Gaussian distribution has been the favorite noise model commonly employed in radio communications mainly because it often leads to closed-form solutions and to linear processors. However, in wireless channels, multiuser interference, atmospheric noise (thunderstorms), car ignitions, and other types of naturally occurring or man-made signal sources result in an aggregate noise component that may exhibit high amplitudes for small duration time intervals $[3,9]$. Recent experimental measurements have demonstrated that the ambient channel noise is decidedly non-Gaussian due mostly to impulsive phenomena (see [12] and references therein). It has been shown that electromagnetic noise in urban mobileradio channels is heavy-tailed in nature and can be better modeled by using distributions with algebraic tails rather 
than the Gaussian or other exponentially-tailed distributions $[7,5,6]$.

Detection and estimation algorithms designed under the Gaussian assumption exhibit various degrees of performance degradation, depending on the non-Gaussian nature of the noise. This is due to the lack of robustness of linear and quadratic types of signal processors to many types of non-Gaussian environments [4]. On the other hand, non-Gaussian noise may actually be beneficial to a system's performance if appropriately modeled and treated [12]. For this reason, engineers have recognized the need to use more general and realistic non-Gaussian models and design robust signal processing techniques that take into account the heavy-tail nature of the data.

Recently, a statistical model of heavy-tailed interference, based on the theory of alpha-stable random processes, has been proposed for signal processing applications [7]. The family of alpha-stable distributions arises under very general assumptions and describes a broad class of impulsive interference. It is a parsimonious statistical-physical model defined (in its most general form) by only four parameters that can be efficiently estimated directly from the data. Furthermore, the alphastable model is the only one whose members obey the stability property and the Generalized Central Limit Thenrem.

\section{Problem Statement}

Most of the theoretical work on blind equalizers based on the CM criterion typically exploit higher (than second) order statistics or second-order cyclostationary statistics of the channel output signal. For this reason these methods have focused on the case where the channel noise is assumed to follow the Gaussian model. The Gaussian assumption is frequently motivated because it often leads to mathematically tractable solutions. However, algorithms designed under the Gaussian assumption exhibit various degrees of performance degradation, depending on the non-Gaussian nature of the environment. As we proved in the simulation experiments, for the Constant Modulus Algorithm class, the presence of heavy-tail nature noise, even though not much impulsive, (i.e. realistic near Gaussian values), lead to a total lost of performance if used with methods optima under the Gaussianity assumption.

Indeed, experimental results have been reported where elcctromagnetic noise in urban mobile-radio channels is heavy-tailed in nature and cannot be modeled by means of Gaussian or other exponential-tailed distributions [5, 6].

Hence, there is a need to use more general and realistic non-Gaussian models and design efficient equalization techniques that take into account the possible heavy-tail nature of the data, and simultaneously work well in good Gaussian channels. In modeling this type of signals the symmetric alpha-stable $(S \alpha S)$ distribution provides an attractive theoretical tool. It was proven that under broad conditions, a general class of heavy-tailed noise follows the stable law [7].

The $S \alpha S$ class of distributions is best defined by its characteristic function, depending by three parameters. The characteristic exponent $\alpha$ is the most important parameter of the $S \alpha S$ distribution and it determines the shape of the distribution. The smaller the characteristic exponent $\alpha$ is, the heavier the tails of the alpha-stable density. It is this heavy-tail characteristic that makes the alpha-stable densities appropriate for modeling noise that may be impulsive in nature. We should also note that the stable distribution corresponding to $\alpha=2$ coincides with the Gaussian density.

A main property of such distributions is that all but Gaussian distributions possess finite moments of order $p$ only when $p$ is strictly less than $\alpha: E|X|^{p}<\infty$ for $p<\alpha$. That explains our choice to use the fractional-lower order moments as the only possible tool capable to digitally process the analyzing data.

Our work is devoted to the development of a novel constant modulus method which makes use of temporal filter diversity and array signal processing system for robust performance in the presence of noise environments that can be modeled according to the alpha-stable law.

\subsection{The CM Algorithm: Background}

Let consider a received signal which is processed by a blind equalizer according to a precise cost function. The complex filter output $y(n)$ may be written as

$$
y(n)=\mathbf{x}^{H}(n) \mathbf{w}(n)
$$

where $\mathbf{x}(n)$ is the vector of data in the delay line of a $N$ coefficient complex FIR filter in the case of omnidirectional analysis. If we have an array of antennas, $\mathbf{x}(n)$ will be the phase-shifted version of the $N$ data signals according to the relative incoming directions.

The most famous member of the constant modulus family is the Constant Modulus (CM) algorithm [10, 11], whose cost function is given by

$$
J_{c m}=\frac{1}{4} E\left[\left.|| y(n)\right|^{2}-\left.\delta\right|^{2}\right]
$$

where $\delta$ is the constant modulus value, $p$ and $q$ are two positive integers, and whose update equation is given by

$$
\mathbf{w}(n+1)=\mathbf{w}(n)-\mu \mathbf{x}^{*}(n) y(n)\left(\|y(n)\|^{2}-1\right)
$$


Such method allows a set of characteristics The main characteristic associated with the standard CMA is that it involves fourth-order moments of the signal. In the presence of heavy-tailed noise, the use of second- or higherorder statistics in effect amplifies the noise.

\section{Alpha Stable Distributions}

The class of symmetric alpha-stable distributions is best defined by its characteristic function:

$$
\varphi(\omega)=\exp \left(\jmath \delta \omega-\gamma|\omega|^{\alpha}\right)
$$

where $\alpha$ is the characteristic exponent restricted to the values $0<\alpha \leq 2, \delta(-\infty<\delta<\infty)$ is the location parameter, and $\gamma(\gamma>0)$ is the dispersion of the distribution. The dispersion parameter $\gamma$ determines the spread of the distribution around its location parameter $\delta$, much in the same way that the variance of the Gaussian distribution determines the spread around the mean. The characteristic exponent $\alpha$ is the most important parameter of the $S \alpha S$ distribution and it determines the shape of the distribution. The smaller the characteristic exponent $\alpha$ is, the heavier the tails of the alpha-stable density. It is this heavy-tail characteristic that makes the alpha-stable densities appropriate for modeling noise and interference which are impulsive in nature. We should also note here that the stable distribution corresponding to $\alpha=2$ coincides with the Gaussian density.

An important difference between the Gaussian and the other distributions of the alpha-stable family is that only moments of order less than $\alpha$ exist for the non-Gaussian alpha-stable family members. That is the main reason of the need to change the cost function and the relative recursive equation. We actually did not use the same cost function with $p$ and $q$ fractional because we referred to one fundamental tool used for the $S \alpha S$ distribution represented by the signed power defined as

$$
x^{<p>}=|x|^{p-1} x *
$$

Using the FLOM and the signed power operators, we came up with a new cost function.

\subsection{Fractional Lower Order CM Filter and Array}

We propose a new cost function that points out mainly two aspects. First, we are processing signals with constant modulus modulation, so this characteristic is what wc eventually desire; second, we suppose to have to deal with a larger and more realistic class of noise distributions, which may give origin to some impulsiveness totally disruptive if managed with the standard CMA. What we present, is a modified version of the error function which takcs into account this heavy-tailed nature of the noise, trying to mitigate its impulsiveness by means of fractional lower-order moments. The new cost function has the expression

$$
J_{p, q}^{F L O S-C M}=E\left[\left|\|y(n)\|^{(p-1)} y(n)-\delta y(n)\right|^{q}\right]
$$

where $\delta$ is a $p$ th -1 power version of the constant signal modulus, assuming $p<\alpha$. The pair $(p, q)$ takes fractional values between 0 and $\alpha$, where $\alpha$ is the characteristic exponent of the alpha-stable distribution that best describes the statistics of the noise vector. Furthermore, the product $p \cdot q$ must be less than $\alpha$. The equations we came out is

$$
\begin{aligned}
& \mathrm{w}(n+1)=\mathrm{w}(n)-\mu \nabla_{w^{*}} J_{p q}= \\
& \mathbf{w}(n)-\mu \frac{q}{2}\|\bar{A}\|^{q-2} \mathbf{x}(n)^{*}\left(p\|y(n)\|^{p-1}-1\right) \bar{A} \\
& \bar{A}=y(n)\left(\|y(n)\|^{p-1}-1\right)
\end{aligned}
$$

One difference between the two parallel analysis is the capability to discriminate two lock and capture zones for the temporal system only if the incoming signals are tones. Adversely, in the case of antennas array it is always possible to find a more efficient expression due to the presence of another information term carried out with the signals represented by the angular spatial arrival direction.

\section{Simulations Results}

In this section, we test and validate the new FLOS-CM Icmporal-spatial adaptive algorithms and compare their performance with that of the conventional $\mathrm{CM}$ algorithms in a noisy environment.

For the spatial model, let consider two independent transmitted signals, QPSK modulated, impinging on the array of dimension two.

For the temporal model, let suppose a filter length of two taps, and consider a received signal which is a scalar sum of the direct signal and a delayed version of itself.

The number of snapshots available to the array is $M=$ $100,000, M=500,000$ or $M=1,000,000$ depending on the expected BER and we performed 10 Montecarlo runs. 


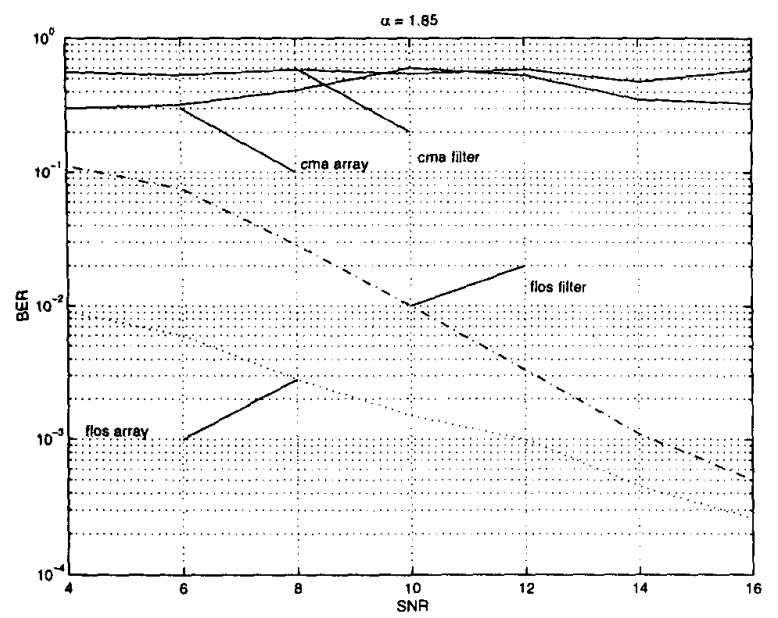

Figure 1: Bit error rate curves for the filter receiver system in configuration for $\alpha=1.85$ and $\alpha=2$.

We model the complex amplitudes of the noise as complex Symmetric-Alpha-Stable random variables. In our simulations, we define the Effective Fractional SNR (EF$S N R$ ) to be the ratio of the fractional signal power of $\alpha$ over the fractional noise power of $\alpha$.

$$
\operatorname{EFSNR}=\sum_{n=1}^{M} \frac{|\mathbf{s}(n)|^{\alpha}}{|\mathbf{n}(n)|^{\alpha}}
$$

We had to use such unusual parameter since the standard SNR, which uses second order powers, is not defined for impulsive noise.

In the Figures 1 and 2 we plot the Bit Error Rate versus the EFSNR for the original CM and the proposed FLOS$\mathrm{CM}$ algorithms for both the filter and the array solutions. In Figure 1 the noise component is modeled as an alphastable process with $\alpha=1.85$. Figure 1 demonstrate that occurrences of noise outliers during the adaptation, have an adverse affect to the learning curve of the original CM method, as the quasi horizontal curves can testify. On the other hand, the proposed FLOS-CM Filter and FLOS-CM Array cost function can suppress the noise components and results in a much deeper bit error rate curve. The difference between the two performance behavior for the FLOS CM filter and array is due to the capability for the array system to opportunely initialize the algorithm in order to obtain a more stable and faster convergence.

In the presence of Gaussian channels, Figure 2, the CM performs better than the FLOS-CM for both the spatial and the temporal models, as we could expect, since the $\mathrm{CM}$ gives the optimum solution.

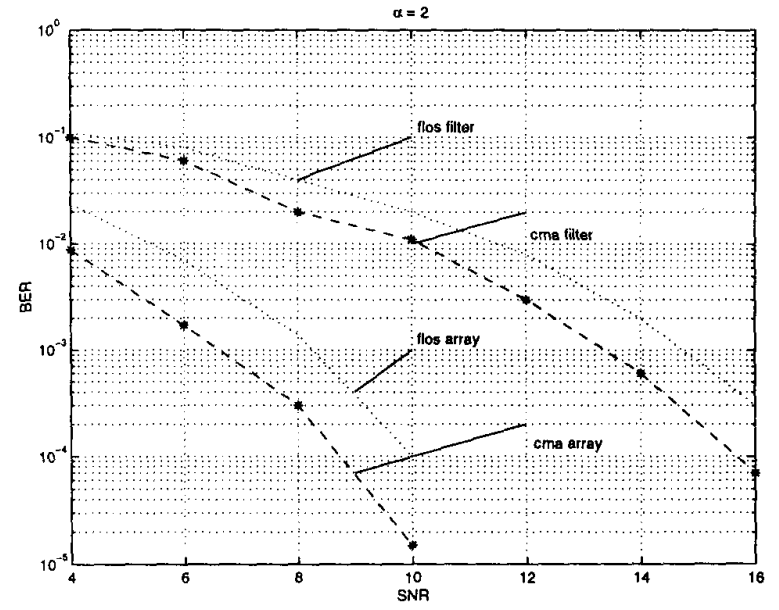

Figure 2: Bit error rate curves for the filter receiver system in configuration for $\alpha=1.85$ and $\alpha=2$.

\section{Conclusions}

In this paper we have developed a new cost function and a new relative update equation for the treatment of constant modulus signals over a heavy-tailed noise channel. The key feature of this new cost function is its robustness and reliability in the presence of different noise environments. The capability of an array system to steer a beam in the direction of the signal while suppressing interference and noise is resulting in a better performance in terms of bit error rate respect to the equivalent FIR temporal filter case.

\section{References}

[1] R. Gooch and J. Lundell al. The cm array: an adaptive beamformed for constant modulus signals. In Proc. of ICASSP 86, 1986.

[2] S Haykin. Adaptive Filter Theory. Prentice-Hall, Englewood Cliffs, NJ, third edition, 1996.

[3] C.L. Nikias I.S. Reed and V. Prasanna. Multidisciplinary research on advanced high-speed, adaptive signal processing for radar sensor. Technical report, University of Southern California, June 1996.

[4] S. A. Kassam and H. V. Poor. Robust techniques for signal processing: A survey. Proc. of the IEEE, 73:433-481, Mar. 1985 .

[5] D. Middleton. Statistical-phisical models of urban radio noise environment - part i: Foundations. IEEE Trans. Elect. Comput., 14:38-56, 1972. 
161 U. Mitra and H.V. Poor. Detection of spreadspectrum signals in multi-user environment. In Proc. of IEEE ICASSP, pages 1844-1847, Detroit, Michigan, May 9-12, 1995.

[7] C L Nikias and M Shao. Signal Processing with Alpha-Stable Distributions and Applications. John Wiley and Sons. New York, 1995.

[8] J.G. Proakis. Digital Communications. McGrawHill, New York, 1995.

[9] B.W. Stuck and B. Kleiner. A statistical analysis of telephone noise. Technical report. Bell. Syst. Tech. J., June 1974.

[10] J.R. Treichler and B.G. Agee. A new approach to multipath correction of constant modulus signals. IEEE Transactions on Acoustic, speech, and Signal Processing, Apr. 1983.

[11] J.R. Treichler and M.G. Larimore. New processing techniques based on the constant modulus adaptive ãlgorithm. IEEE Transactions on Acoustic, Speech, and Signal Processing, Apr. 1985.

[12] X. Wang and H. V. Poor. Robust multiuscr detection in non-gaussian channels. IEEE Trans. on Signal Processing, 47:289-305, Feb. 1999. 